\title{
Apparent Intravoxel Fibre Population Dispersion (FPD) Using Spherical Harmonics
}

\author{
Haz-Edine Assemlal ${ }^{1}$, Jennifer Campbell ${ }^{2}$, Bruce Pike ${ }^{2}$, and Kaleem Siddiqi ${ }^{1}$ \\ 1 Centre for Intelligent Machines, McGill University, \\ 3480 University Street, Montréal, QC, Canada H3A 2A7 \\ 2 McConnell Brain Imaging Centre, Montreal Neurological Institute, \\ 3801 University Street, Montréal, QC Canada H3A 2B4 \\ assemlal@cim.mcgill.ca
}

\begin{abstract}
The vast majority of High Angular Resolution Diffusion Imaging (HARDI) modeling methods recover networks of neuronal fibres, using a heuristic extraction of their local orientation. In this paper, we present a method for computing the apparent intravoxel Fibre Population Dispersion (FPD), which conveys the manner in which distinct fibre populations are partitioned within the same voxel. We provide a statistical analysis, without any prior assumptions on the number or size of these fibre populations, using an analytical formulation of the diffusion signal autocorrelation function in the spherical harmonics basis. We also propose to extract features of the FPD obtained in the group of rotations, using several metrics based on unit quaternions. We show results on simulated data and on physical phantoms, that demonstrate the effectiveness of the FPD to reveal regions with crossing tracts, in contrast to the standard anisotropy measures.
\end{abstract}

\section{Introduction}

Diffusion magnetic resonance imaging (dMRI) allows one to examine the microscopic diffusion of water molecules in biological tissue in-vivo. In practice, this imaging modality requires the collection of successive images with magnetic field gradients applied in different directions [1. A reconstruction step is then used to estimate the 3D diffusion probability density function (PDF) from the acquired images 2]. Recently a Spherical Polar Fourier (SPF) expansion method has been introduced in [3], which takes full advantage of the acquisition protocol. Here the MR signal attenuation $E$ at the diffusion wave-vector $\mathbf{q}$ of the $q$-space (a subset of Euclidean $\mathbf{R}^{3}$ ) is expressed as the following series in the SPF basis:

$$
E(\mathbf{q})=\sum_{n, l, m \in \mathbf{D}} a_{n l m} \sqrt{\frac{2}{\zeta^{3 / 2}} \frac{n !}{\Gamma(n+3 / 2)}} \exp \left(-\frac{q^{2}}{2 \zeta}\right) L_{n}^{1 / 2}\left(\frac{q^{2}}{\zeta}\right) y_{l}^{m}(\hat{\mathbf{q}}),
$$

where the triplet $\{n, l, m\}$ stands for the index defined in the set $\mathbf{D} \in \mathbf{N}^{2} \times \mathbf{Z}$ so that $n \in \mathbf{N}$ is the radial index, and $l \in \mathbf{N}, m \in \mathbf{Z},-l \leq m \leq l$ are the angular indexes. The symbols $a_{n l m}$ are the series coefficients, $y_{l}^{m}$ are the real 
spherical harmonics $(\mathrm{SH})$, and $R_{n}$ is an orthonormal radial basis function made of Gaussian-Laguerre (GL) functions.

Let $L^{2}\left(\mathbf{S}^{2}\right)$ denote the space of square integrable functions on $\mathbf{S}^{2}$. It has been shown in [3] that any feature $f \in L^{2}\left(\mathbf{S}^{2}\right)$ of the diffusion PDF can be directly extracted from the modeled diffusion signal in the SPF expansion [3] as

$$
f(\hat{\mathbf{r}})=\sum_{n, l, m \in \mathbf{D}} a_{n l m} b_{n l m} y_{l}^{m}(\hat{\mathbf{r}})=\sum_{n, l, m \in \mathbf{D}} f_{n l m} y_{l}^{m}(\hat{\mathbf{r}})
$$

where the symbol $\hat{\mathbf{r}}$ is a unit vector in the 2-sphere $\mathbf{S}^{2}, b_{n l m}$ stands for the coefficients of the feature projection function expressed in the SPF expansion and $f_{n l m}=a_{n l m} b_{n l m}$ are the spherical harmonics coefficients of $f$. The feature related to the second-order Orientation Density Function $\left(\mathrm{ODF}_{2}\right)$ displacement of water molecules is expressed in the SPF basis as [4]:

$$
b_{n l m}=\frac{\delta_{l 0} \delta_{m 0}}{\sqrt{4 \pi}}-\frac{1}{8 \pi} \sum_{j=0}^{n} \frac{(-1)^{j}}{j 2^{j}} \sqrt{\frac{2}{\zeta^{3 / 2}} \frac{n !}{\Gamma(n+3 / 2)}}\left(\begin{array}{c}
n+1 / 2 \\
n-j
\end{array}\right) P_{l}(0)\left(-l^{2}+l\right) .
$$

Other spherical features of the diffusion PDF are given in [3, 4. Given a spherical feature $f \in L^{2}\left(\mathbf{S}^{2}\right)$ such as (3), which represents the orientation features of the diffusion PDF, this paper presents a method to compute the statistical dispersion of fibre populations within a voxel, without any prior assumptions on their number or shape (Section 2). We illustrate the validity of the proposed method on synthetic and physical phantom datasets (Section 3 ).

Among the previous related works, Seunarine et al. proposed in [5] to compute the anisotropy of each fibre population within the same voxel. Other relevant methods include the labelling of crossing and fanning fibre populations [6] and the computation of a torsion index [7, both using inter-voxel computations. To the extent of our knowledge, the present paper describes the first statistical method for assessing the partitioning of fibre populations within the same voxel. As such, it may have a significant impact in applications involving the study of neurological diseases from dMRI.

\section{Theory}

\subsection{Fibre Population Dispersion (FPD)}

Let $\mathbf{S O}(\mathbf{3})$ denote the rotation group. According to Euler's rotation theorem, any rotation $\Lambda \in \mathbf{S O}(3)$ can be described by three successive rotations by a set of Euler angles $\boldsymbol{\Theta}=(\alpha, \beta, \gamma)$ about three axes, where $0 \leq \alpha, \gamma \leq 2 \pi$ and $0 \leq \beta \leq \pi$. There are 24 standard Euler angle conventions depending upon which axes are used and the order in which the rotations are applied. Throughout this paper, we use the $z y z$-configuration to compose the intrinsic rotations. In this setting, we can parametrize the general rotation $\Lambda$ as a function of the set of Euler angles $\boldsymbol{\Theta}$. 


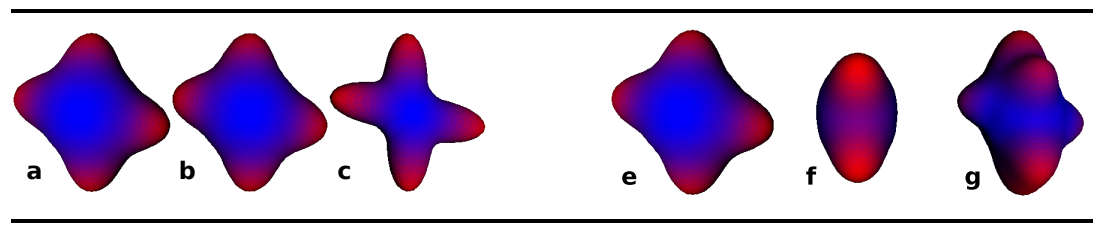

Fig. 1. The Fibre Population Dispersion (FPD) is defined as the autocorrelation of any spherical feature function defined on $\mathbf{S}^{2}$ of the diffusion PDF. Left: (a) Two-dimensional projection of the $\mathrm{ODF}_{2}$ for two fibre populations crossing at $1.37 \mathrm{rad}$ in the $x y$-plane. (b) $\mathbf{S O}(3)$ rotation of (a) by the set of Euler angles $\boldsymbol{\Theta}_{1}$. (c) The point-wise $\mathbf{S}^{2}$ multiplication of (a) and (b), from which the autocorrelation is computed as the integral volume. Right: The same computations, but with a different set of Euler angles $\boldsymbol{\Theta}_{2}$. The FPD of (c) is greater than the FPD of $(\mathrm{g})$, with a ratio equal to 1.14 , so that the rotation relates to the distance between intravoxel fibre populations as a function of Euler angles $\Theta$. Note that in this figure, the functions are scaled for visualization purposes.

Let $\Lambda(\boldsymbol{\Theta}) f(\hat{\mathbf{r}})$ denote a rotation $\Lambda$ of the function $f \in L^{2}\left(\mathbf{S}^{2}\right)$ such that it is equivalent to $f\left(\Lambda^{-1}(\boldsymbol{\Theta}) \hat{\mathbf{r}}\right)$. We define the Fibre Population Dispersion (FPD) as the normalized autocorrelation of the spherical feature function $f \in L^{2}\left(\mathbf{S}^{2}\right)$, so that $\mathrm{FPD}: \mathbf{S O}(\mathbf{3}) \rightarrow \mathbf{R}$ and

$$
\operatorname{FPD}(\Lambda)=\int_{\hat{\mathbf{r}} \in \mathbf{S}^{2}} \frac{(f(\hat{\mathbf{r}})-\bar{f})}{\sigma_{f}} \frac{\Lambda(\boldsymbol{\Theta})(f(\hat{\mathbf{r}})-\bar{f})}{\sigma_{r}} \mathrm{~d} \hat{\mathbf{r}}
$$

where $\sigma_{f}$ and $\sigma_{r}$ denote the standard variation of $f$ and its rotated version $\Lambda(\Theta) f$. Fig. 1 clarifies the intuition behind (4), i.e., how the correlation function between a spherical feature and a rotated version of it can recover fibre population dispersion. The left and right parts of Fig. 1 illustrate high and low values of the FPD, respectively.

Since the function $f$ and its rotated version $\Lambda(\boldsymbol{\Theta})(f)$ have the same standard variations, the normalizing factor is therefore the variance of $f$, i.e., $\sigma_{f}^{2}$. Let $\mathbf{D}^{*}=\mathbf{D} \backslash\{l=0\}$ be the index domain minus all the terms which nullify the order $l$. Fortunately, the variance and the mean are directly expressed in the spherical harmonics space, which yields the following simplification of (4):

$$
\operatorname{FPD}(\Lambda)=N \int_{\hat{\mathbf{r}} \in \mathbf{S}^{2}} \sum_{n, l, m \in \mathbf{D}^{*}} f_{n l m} y_{l}^{m}(\hat{\mathbf{r}}) \Lambda(\boldsymbol{\Theta}) \sum_{i, j, k \in \mathbf{D}^{*}} f_{i j k} y_{j}^{k}(\hat{\mathbf{r}}) \mathrm{d} \hat{\mathbf{r}},
$$

where the normalization factor $N$ is obtained by inverting the sum of square coefficients $f_{n l m}$. When subject to a rotation, real spherical harmonics $y_{l}^{m}$ can be expressed as a linear combination of real spherical harmonics of the same order $l[8$. As a consequence, (5]) can be expressed as

$$
\operatorname{FPD}(\Lambda)=N \int_{\hat{\mathbf{r}} \in \mathbf{S}^{2}} \sum_{n, l, m \in \mathbf{D}^{*}} f_{n l m} y_{l}^{m}(\hat{\mathbf{r}}) \sum_{i, j, k \in \mathbf{D}^{*}} f_{i j k} \sum_{k^{\prime}=-j}^{j} R_{k^{\prime} k}^{(j)}(\boldsymbol{\Theta}) y_{j}^{k^{\prime}}(\hat{\mathbf{r}}) \mathrm{d} \hat{\mathbf{r}},
$$


where $R_{k^{\prime} k}^{(j)}(\boldsymbol{\Theta})$ are the real Wigner-D functions. On integrating over the unit sphere, all terms vanish except $n=i, l=j$ and $m=k^{\prime}$ to give an expression for the FPD at rotation $\Lambda$ :

$$
\operatorname{FPD}(\Lambda)=\left(\sum_{n, l, m \in \mathbf{D}^{*}} f_{n l m}^{2}\right)^{-1} \sum_{n, l, m \in \mathbf{D}^{*}} f_{n l m} \sum_{k=-l}^{l} f_{n l k} R_{k m}^{(l)}(\boldsymbol{\Theta}),
$$

in which the normalization factor $N$ has been replaced by its expression. It is remarkable that (7) can be interpreted as a result of the Wiener-Khinchin theorem which states that the autocorrelation of $f$ is the inverse $\mathbf{S O}(\mathbf{3})$-Fourier transform of the corresponding power spectral density function.

The expression in (7) is a closed-form solution of (4), which directly relates the FPD to the SPF expansion coefficients of $f$ for a single voxel. This avoids the need for cumbersome numerical discretization schemes of $f$ on the 2-sphere for each voxel, by turning the FPD computation into a fast dot product between vectors of SPF coefficients. Note that the result obtained in (7) is not only valid for the Spherical Polar Fourier (SPF) expansion method [3] but also for any local reconstruction method of the diffusion signal based on spherical harmonics functions (e.g., Q-Ball Imaging (QBI) 9], the Diffusion Orientation Transform (DOT) [10] and Diffusion Propagator Imaging (DPI) [11).

\section{$2.2 \quad$ A Distance Metric}

Having meaningful statistics on the FPD function $\mathbf{S O}(\mathbf{3}) \rightarrow \mathbf{R}$ given in (7) requires the definition of a distance metric on the rotation group $\mathbf{S O}(3)$. Although it is possible to define a metric which uses the Euler angles, such a parametrization will degenerate at some points on the hypersphere, leading to the problem of gimbal lock, the loss of one degree of rotational freedom. We avoid this by using the quaternion representation $\mathbf{h} \in \mathbf{H} \subset \mathbf{R}^{4}$, i.e., a set of four Euclidean coordinates $\mathbf{h}=\{(a, b, c, d) \mid a, b, c, d \in \mathbf{R}\}$ using the basis elements $1, i, j, k$ which satisfy the relationship $i^{2}=j^{2}=k^{2}=i j k=-1$. Quaternions can parametrize rotations in $\mathbf{S O}(\mathbf{3})$ by constraining their norm to unity, i.e., $\|\mathbf{h}\|=a^{2}+b^{2}+c^{2}+d^{2}=1$. In this setting, the four scalar numbers have only three degrees of freedom and the unit quaternions form a subset $\mathbf{S}^{3} \subset \mathbf{R}^{4}$. If we regard the quaternions as elements of $\mathbf{R}^{4}$, any usual $L_{p}$ norm $\rho_{s}$ can be used to define a metric on $\mathbf{S O}(\mathbf{3})$ between $\mathbf{h}_{1}$ and $\mathbf{h}_{2}$ :

$$
\rho\left(\mathbf{h}_{1}, \mathbf{h}_{2}\right)=\min \left\{\rho_{s}\left(\mathbf{h}_{1}, \mathbf{h}_{2}\right), \rho_{s}\left(\mathbf{h}_{1},-\mathbf{h}_{2}\right)\right\},
$$

Throughout this paper, $\rho_{s}$ stands for the $L_{2}$ norm. It follows that $(\mathbf{H}, \rho)$ is a metric space. We define the $p$-th moment of the FPD about a given quaternion $\mathbf{h}_{0} \in \mathbf{H}$ as

$$
\mathcal{M}_{p}\left(\mathbf{h}_{o}\right)=\int_{\mathbf{h} \in \mathbf{H}} \rho\left(\mathbf{h}, \mathbf{h}_{0}\right)^{p} d \operatorname{FPD}(\mathbf{h})
$$



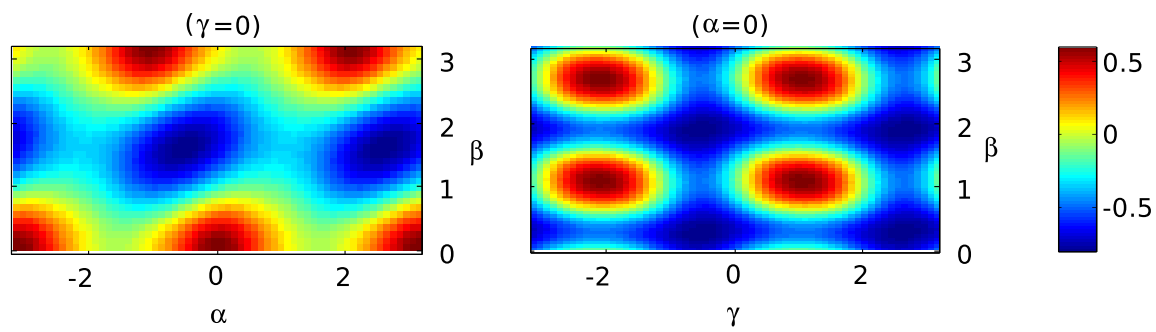

Fig. 2. The Fibre Population Dispersion (FPD) function reveals the autocorrelation of a spherical feature of the diffusion PDF. The FPD is computed on the $\mathrm{ODF}_{2}$ composed of two fibre populations crossing at $0.5 \mathrm{rad}$ in the $x y$-plane. Slices of the threedimensional FPD are are plotted in this figure on a Cartesian grid by selecting a uniform sampling in Euler angles sets $(\alpha, \beta, \gamma)$, with $d \alpha=d \beta=d \gamma=0.1 \mathrm{rad}$ (see text for a discussion).

with $p \in \mathbf{N}$. The $p$-th moments of the FPD defined in (9) give a quantitative measure of the shape of the FPD, and yield different values depending on the separation, size and numbers of fibre populations (as discussed further in Section 3). The moment $\mathcal{M}_{p}$ can be computed about any unit quaternion $\mathbf{h}_{0} \in \mathbf{H}$, but the identity quaternion $\mathbf{h}_{I}=k$ represents a zero rotation and is consequently the most "natural" choice in the sense that it leads to the central moments $\mathcal{M}_{p}\left(\mathbf{h}_{I}\right)$.

\section{Results}

\subsection{Synthetic Data}

Fig. 2 illustrates a simulated diffusion PDF for two fibre populations crossing in the same voxel (left of Fig. 2) and its related Fibre Population Dispersion (FPD) function computed using the closed-form we provide in (7). We chose the Euler $z y z$-convention, therefore the symbol $\alpha$ denotes the Euler angle around the $z$-axis, $\beta$ the angle around the rotated $y$-axis and $\gamma$ around the twice-rotated $z$-axis (right of Fig. 2). Note that although apparent proximity or distance may appear in the FPD image of Fig. 2, this may be misleading because the Euler angles do not form an Euclidean metric. This results in a distorted mapping of the neighbourhood compared to a proper metric such as the quaternion distance $\rho\left(\mathbf{h}_{1}, \mathbf{h}_{2}\right)$ (8) . Nonetheless, it is relatively easy to identify the periodicity of the FPD values of fibre populations which are conveniently oriented according to the Euler axis of rotations. In Fig. 2, the variations of the FPD function along $\beta$ values on the line defined by $\gamma=1.1 \mathrm{rad}$ and $\alpha=0 \mathrm{rad}$ reveals the presence of two fibre populations at $\beta=\{1.1,2.6\} \mathrm{rad}$.

The computation of the closed-form FPD expression given in (7) requires one to convert unit quaternions to Euler angles. A very efficient and generic conversion is given in [12]. 
Table 1. MICCAI 2009 Fibre Cup Phantom: parameters of the diffusion acquisition sequence [13. The diffusion sensitization was applied along a set of 64 orientations.

\begin{tabular}{ccc}
\hline Scanner Siemens TrioTim $(12$ channel $)$ & Sequence=SSTR & Magnetic field=3 T \\
Voxel size $=3 \times 3 \times 3 \mathrm{~mm}^{3}$ & Image size $=64 \times 64 \times 3$ & TR $=5000 \mathrm{~ms}$ \\
$b$-value $=\{650,1500,2000\} \mathrm{s} \mathrm{mm}^{-2}$ & $\|\mathbf{g}\|_{\max }=40 \mathrm{mT} \mathrm{m}^{-1}$ & $\mathrm{TE}=\{77,94,102\} \mathrm{ms}$ \\
\hline
\end{tabular}

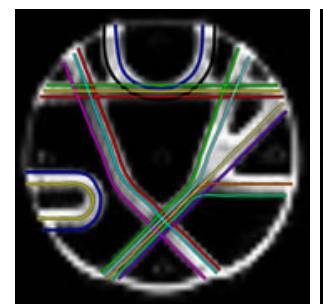

(a) Ground Truth (left)

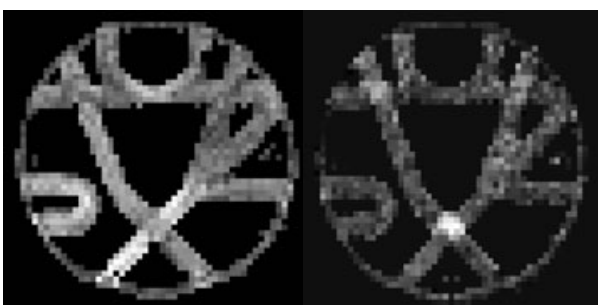

(b) GFA [14.

(c) FPD (our result).

Fig. 3. The Fibre Cup phantom consists of fibre bundles crossing at specific locations across the slice. We show a comparison of the generalized fractional anisotropy (GFA) measure and our fibre population dispersion (FPD) measure. The GFA image does not explicitly reveal crossings, while in the FPD image the brightness of a voxel is proportional to the crossing angle.

\subsection{MICCAI 2009: Fibre Cup Phantom}

For evaluating our proposed analytical computation of the fibre population dispersion (FPD), we present results on the MICCAI 2009 Fibre Cup phantom. It is composed of large bundles made of hydrophobic acrylic fibres whose diameter is of the same order of that of myelinated axons, with a density close to 1900 fibres $/ \mathrm{mm}^{2}$. The parameters of the acquisition are described in Table 1 and the phantom is shown in Fig. 33. The sampling uses three spheres in the $q$-space, each having a $q$ radius proportional to the $b$ value, so that $b=(2 \pi)^{2}(\Delta-\delta / 3) q^{2}$. The SPF reconstruction technique [3] is able to naturally take advantage of the full set of samples provided, unlike HARDI reconstruction techniques which are restricted to a subset of samples (i.e., a single sphere in the $q$-space). It was also recently demonstrated to lead to more accurate and robust reconstruction of the diffusion signal [4.

Fig. 3 illustrates the computation of the FPD on this phantom. As can be seen in the ground truth image (c.f., Fig. 3a), there are a limited number of crossings located at specific regions of interest (ROIs) named (a-f). This controlled environment is ideal to assess the potential of the FPD. Furthermore, the ROIs can be sorted by the angle of the crossing, from nearly $\pi$ rad at ROI (a) to 
Table 2. Rat spinal cord phantom: parameters of the diffusion acquisition sequence [15]. The diffusion sensitization was applied along a set of 90 orientations.

\begin{tabular}{ccc}
\hline Scanner Siemens Sonata & Sequence $=$ SSTR & Magnetic field $=1.5 \mathrm{~T}$ \\
Voxel size $=2.5 \times 2.5 \times 2.5 \mathrm{~mm}^{3}$ & Image size $=128 \times 96 \times 40$ & $\mathrm{TR}=8000 \mathrm{~ms}$ \\
$b$-value $=\{3000\} \mathrm{s} \mathrm{mm}^{-2}$ & Acquisition time $=15 \mathrm{~min}$ & $\mathrm{TE}=\{110\} \mathrm{ms}$ \\
\hline
\end{tabular}

fanning fibres at ROIs (e,f). Fig. 3b shows the widely used generalized fractional anisotropy (GFA) measure [14, which reflects the degree of angular coherency but does not immediately reveal ROIs corresponding to crossings. In contrast, our FPD technique, with the moment $\mathcal{M}_{8}$ measure proposed in (9), brings to light these ROIs with brightness levels that depend on the angle of crossing, as illustrated in Fig. 3r. In our experiments, the moment $p$-th order is based on heuristics with a balance between good contrast for high moment order $p$ and robustness to low signal-to-noise ratio for low order $p$. Our results indicates that $p=2$ offers a good balance.

\subsection{Rat Spinal Cord Phantom}

For further evaluation of our FPD technique with HARDI data, Fig. 4 shows results computed on a biological phantom constructed from excised rat spinal cords with known connectivity 15. Fig. 4a demonstrates that this biological phantom possesses a single region of crossing tracts. As before, we compare the GFA measure and our FPD technique using the moment $\mathcal{M}_{6}$.

The central voxel of the phantom interestingly exhibits a three dimensional crossing, as a result of one fibre curving over the other. We hypothesize that this diffusion profile results from the averaging of both tracts in a single voxel. Conse-

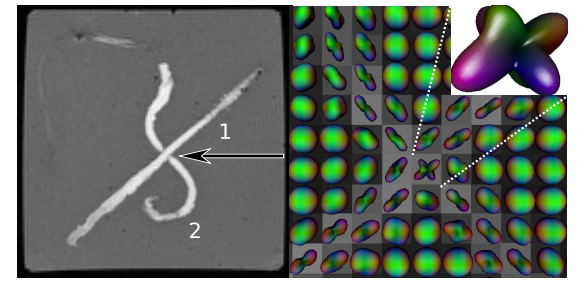

(a) T1-Weighted image (left) and the isoprobability feature 3] (right).

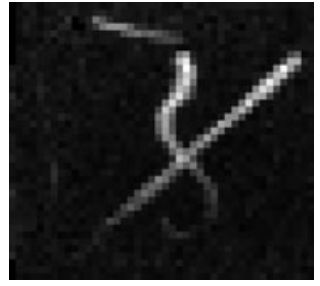

(b) GFA [14].

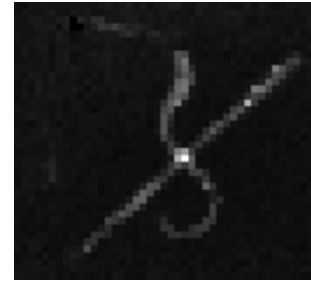

(c) FPD (our result).

Fig. 4. Rat spinal cord phantom: it is constructed from excised rat spinal cords embedded in $2 \%$ agar [15]. (a): an image of the phantom, and a zoom-in on the crossing. (b-c): comparison of the generalized fractional anisotropy (GFA) measure and our fibre population dispersion (FPD) measure. The latter clearly displays a single crossing region, in correspondance with the ground truth. 
quently, this yields the highest FPD value in the slice, as shown in Fig. 4, whereas the GFA does not identify this region. The remaining parts of the rat spinal cords have a relatively constant brightness, which is consistent with the fact that their diffusion profiles are very similar, except for the preferred direction of diffusivity.

\section{Conclusion}

In this paper we have presented a novel method which uses images acquired with dMRI to statistically assess the intravoxel angular dispersion of fibre populations (FPD). We have demonstrated a closed-form expression for any spherical function based on the diffusion Probability Function (PDF), and expressed in the spherical harmonics basis. We have provided a proper metric on the rotation group for analyzing the FPD to obtain the relative distance between intravoxel fibre populations. This naturally leads to the introduction of scalar indices such as the moments of the FPD, which summarizes the intra-voxel dispersion of fibres. The proposed approach is based on a local per-voxel statistical analysis, and is thus fundamentally different from non-local techniques such as tractography. We have illustrated the potential of the FPD technique on both synthetic data and physical phantoms. Our experiments reveal features of the underlying microstructure, and in particular, reveal crossing regions that are not discernible in the widely used GFA images. As such, the FPD may yield interesting new possibilities for the detection and monitoring of neurological disease from dMRI.

\section{References}

1. Stejskal, E., Tanner, J.: Spin diffusion measurements: spin echoes in the presence of a time-dependent field gradient. J. Chem. Phys. 42, 288-292 (1965)

2. Stejskal, E.: Use of spin echoes in a pulsed magnetic-field gradient to study anisotropic, restricted diffusion and flow. J. Chem. Phys. 43(10), 3597-3603 (1965)

3. Assemlal, H.E., Tschumperlé, D., Brun, L.: Efficient and robust computation of PDF features from diffusion MR signal. Med. Image Anal. 13(5), 715-729 (2009)

4. Cheng, J., Ghosh, A., Deriche, R., Jiang, T.: Model-free, regularized, fast, and robust analytical orientation distribution function estimation. In: Jiang, T., Navab, N., Pluim, J.P.W., Viergever, M.A. (eds.) MICCAI 2010. LNCS, vol. 6361, pp. 648656. Springer, Heidelberg (2010)

5. Seunarine, K.K., Cook, P.A., Hall, M.G., Embleton, K.V., Parker, G.J.M., Alexander, D.C.: Exploiting peak anisotropy for tracking through complex structures. In: ICCV, pp. 1-8. IEEE, Los Alamitos (2007)

6. Savadjiev, P., Campbell, J., Descoteaux, M., Deriche, R., Pike, G., Siddiqi, K.: Labeling of ambiguous subvoxel fibre bundle configurations in high angular resolution diffusion MRI. NeuroImage 41(1), 58-68 (2008)

7. Savadjiev, P., Kindlmann, G., Bouix, S., Shenton, M., Westin, C.: Local white matter geometry from diffusion tensor gradients. NeuroImage 49(4), 3175-3186 (2010)

8. Su, Z., Coppens, P.: Rotation of real spherical harmonics. Found. Cryst. 50(5), 7673 (1994) 
9. Anderson, A.: Measurement of fiber orientation distributions using high angular resolution diffusion imaging. Magn. Reson. Med. 54(5) (2005)

10. Özarslan, E., Sherperd, T.M., Vemuri, B.C., Blackband, S.J., Mareci, T.H.: Resolution of complex tissue microarchitecture using the diffusion orientation transform (DOT). NeuroImage 31, 1086-1103 (2006)

11. Descoteaux, M., Deriche, R., Bihan, D., Mangin, J., Poupon, C.: Multiple q-Shell Diffusion Propagator Imaging. Med. Image Anal. (2010)

12. Shoemake, K.: Graphics gems IV. In: Euler Angle Conversion, pp. 222-229. Academic Press Professional, Inc., London (1994)

13. Poupon, C., Rieul, B., Kezele, I., Perrin, M., Poupon, F., Mangin, J.F.: New diffusion phantoms dedicated to the study and validation of high-angular-resolution diffusion imaging (HARDI) models. Magn. Reson. Med. 60(6), 1276-1283 (2008)

14. Tuch, D.: Q-ball imaging. Magnetic Resonance in Medicine 52, 1358-1372 (2004)

15. Campbell, J., Siddiqi, K., Rymar, V., Sadikot, A., Pike, G.: Flow-based fiber tracking with diffusion tensor and q-ball data: validation and comparison to principal diffusion direction techniques. NeuroImage 27(4), 725-736 (2005) 